\title{
Accommodation, pattern glare, and coloured overlays
}

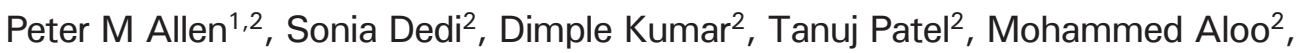 \\ Arnold J Wilkins ${ }^{3}$ \\ ${ }^{1}$ Vision and Eye Research Unit, Postgraduate Medical Institute, Cambridge CB1 1PT, UK; \\ ${ }^{2}$ Department of Vision and Hearing Sciences, Anglia Ruskin University, East Road, \\ Cambridge CB1 1PT, UK; e-mail: peter.allen@anglia.ac.uk; ${ }^{3}$ Visual Perception Unit, \\ Department of Psychology, University of Essex, Colchester CO4 3SQ, UK \\ Received 4 October 2012, in revised form 20 November 2012
}

\begin{abstract}
We manipulated the accommodative response using positive and negative lenses to study any association between symptoms of pattern glare and accommodation. Two groups of eighteen young adults were selected from seventy-eight on the basis (i) that their rate of reading increased by $5 \%$ or more with an overlay compared to their rate without it, and (ii) that they reported more than 2 symptoms of pattern glare (group 1) or had no such increment in reading speed and reported fewer than 3 symptoms (group 2). Under double-masked conditions participants observed at $0.4 \mathrm{~m}$ a pattern of stripes while measurements of accommodation were made using an open field autorefractor with and without positive and negative trial lenses $(0.75 \mathrm{D})$, and with and without a coloured overlay. Pattern glare was also assessed with and without the trial lenses. Without lenses, the mean accommodative response in group 1 was $1.55 \mathrm{D}$, a lag of $0.95 \mathrm{D} \pm 0.24 \mathrm{D}$ relative to the demand. The lag decreased by $0.43 \mathrm{D}(p<0.0001)$ when the chosen overlay was used, an effect that was not shown in group 2 even when lag increased with negative trial lenses $(p=0.13)$. In both groups, pattern glare scores were reduced by the trial lenses, but were unaffected by the sign of the lenses. This suggests that symptoms of pattern glare are not strongly associated with accommodative response.
\end{abstract}

Keywords: accommodation, pattern glare, pattern-related visual stress, colour

\section{Introduction}

When people read, an accommodative lag (or under-accommodation relative to the near stimulus) of up to $0.50 \mathrm{D}$ is expected (Rouse et al 1984), but the text will remain clear provided the accommodative error lies within the depth of focus of the eye, which shows individual variation (Atchison et al 1997). Inappropriate accommodative responses, such as under-accommodation or over-accommodation relative to the plane of the object of regard are a frequent correlate of aesthenopia (Allen et al 2010a).

Previous work has inconsistently found increased accommodative microfluctuations in a small sample of individuals who benefit from coloured filters (Simmers et al 2001; Allen et al 2010b), a significant positive correlation between accommodative lag (Chase et al 2009), and symptoms of visual discomfort with near work, and lags of accommodation that were well outside the depth of focus with near targets of $4 \mathrm{D}$ or more for students with moderate or severe symptoms of visual discomfort (Tosha et al 2009). On the other hand, Ciuffreda et al (1997) found no significant differences in the accommodative responses in a small group of Irlen lens wearers with and without their coloured lenses.

Allen et al (2010b) demonstrated that accommodative lag was greater in individuals susceptible to pattern-related visual stress (PRVS), as classified by both (i) a susceptibility to pattern glare and (ii) an increment in reading speed from the use of coloured filters. The Pattern Glare Test assesses the illusions of colour, shape, and motion induced when viewing three gratings of differing spatial frequencies: low (pattern 1), medium (pattern 2), and high (pattern 3). A normative study of the Pattern Glare Test showed that people have an abnormal 
degree of pattern glare if they achieve a score of $>3$ on the medium grating (spatial frequency of 3 cycles deg ${ }^{-1}$; Evans and Stevenson 2008). The illusions of colour, shape, and motion are thought to be at least partly neurological in origin and have been shown to relate to headaches in a variety of ways (Wilkins et al 1984; Nulty et al 1987; Harle et al 2006). Responses to the Pattern Glare Test predict the increase in reading speed with an overlay of chosen colour (Hollis and Allen 2006). A coloured background, similar in colour to that chosen by the participant to aid comfort when viewing text, reduced the lag of accommodation in the PRVS group but made no difference or increased the accommodative lag in the control groups. However, the PRVS group had a much greater lag of accommodation than the control groups even after the lag was reduced with the coloured background.

One interpretation of the above findings is that the use of a coloured filter resulted in a greater effort at accommodation (an increased voluntary accommodative response), perhaps as a result of greater comfort. The objectives of the current study were to manipulate the accommodative response optically with positive and negative trial lenses, and by manipulating accommodative demand assess any differences between groups with respect to the effort individuals are prepared to expend in reducing blur. The requirement for a greater voluntary accommodative response can be reduced by reducing accommodative demand with positive lenses. If the differences observed by Allen et al (2010b) were the result of an increased voluntary accommodative response, any effect of the coloured filter on accommodation should also be reduced. Moreover, the differences in accommodative lag between the PRVS group and controls without coloured filters were $0.39 \mathrm{D}$ on average. It should therefore be instructive to investigate the effects of filters in a control group in which accommodative demand is increased with negative lenses with a possible associated increase in accommodative effort. The manipulation of accommodation also permits an evaluation of the effects of accommodative demand on pattern glare, which may in turn influence accommodative effort.

If any effect of coloured filters on accommodation is mediated by the blur that results from longitudinal chromatic aberration (Chase et al 2007), then a relationship between the chosen colour and the effects of trial lens should emerge.

\section{Methods}

The participants were recruited, by advertisement, from the student population attending Anglia Ruskin University. All participants gave informed consent following a written and verbal explanation of the procedures involved. All procedures conformed to the tenets of the Declaration of Helsinki and were approved by the Anglia Ruskin University Ethics Committee.

\subsection{Session 1: Screening and classification for PRVS and control group participants}

Eighty young adults (forty-six females and thirty-four males aged between 18 and 26 years) attended an initial screening session to exclude any participants with migraine headache or aura, a history of epilepsy, a diagnosis of autism, and/or significant optometric and binocular vision anomalies. Symptoms described by persons suffering from PRVS such as headaches, blurring, and words moving on the page are non-specific and may also be caused by refractive error or binocular anomalies, and these were assessed. The inclusion criteria are shown in table 1. The instructions for the Mallett fixation disparity test were similar to those used by Karania and Evans (2006). They were: "Can you see the circle with the four red lines and the cross" and "are each of them lined up with the cross?" Binocular instability was not assessed in this study. Of the eighty, seventy-eight satisfied all inclusion criteria listed.

In addition to the above tests, all persons meeting the initial inclusion criteria had an objective assessment of their refractive error using a Nidek AR-600A autorefractor (Allen et al 2003) and their susceptibility to PRVS was assessed using both (a) the Pattern Glare Test and (b) overlay assessment and administration of the Rate of Reading Test. 
Table 1. Inclusion criteria.

Visual acuity of at least $6 / 6$ in each eye

Cover /uncover test of $<5 \Delta$ horizontal phoria and $<0.5 \Delta$ vertical phoria

No slip evidenced on fixation disparity (Mallett unit)

No diplopia reported during the ocular motility test

Near point of convergence (RAF rule) $\leqslant 10 \mathrm{~cm}$

Amplitude of accommodation (push up RAF rule) normal for age (greater than $10 \mathrm{D}$ )

Stereo acuity (Titmus circles) of $<80 \mathrm{~s}$ of arc

Normal red/green colour vision (Ishihara)

Astigmatism of $<0.75 \mathrm{DC}$

(a) The desk surface was illuminated by the light from a compact fluorescent lamp (Osram Dulux S $11 \mathrm{~W} / 865)$ with a correlated colour temperature of $6500 \mathrm{~K}$. At a distance of $0.4 \mathrm{~m}$, participants were shown pattern 2 of the Pattern Glare Test (Wilkins and Evans 2001) - a grating with square-wave luminance profile, Michelson contrast above 0.9, spatial frequency 2.3 cycles deg $^{-1}$, circular in outline, radius $14.3 \mathrm{deg}$. They were asked a series of 14 questions regarding the perceptual distortions that they experienced whilst continually viewing the pattern, beginning "Looking into the centre of the grid that is in front of you .... do you see any of the following? Please answer each question with either yes/no." This was followed by the following list: "pain?; discomfort?; shadowy shapes amongst the lines?; shimmering of the lines?; flickering?; red?; green?; blue?; yellow?; blur?; bending of the lines?; nausea?; dizziness?; unease?". (Note that this list was used by Hollis and Allen (2006) and includes the following symptoms additional to those used by Evans and Stevenson (2008): pain?; discomfort?; red?; green?; blue?; yellow? (rather than just colour); nausea?; dizziness?; unease?) For every 'yes' answer given, one was added to the participant's score. This measure has been shown to be a significant predictor of whether a person will read more quickly with coloured overlays (Hollis and Allen 2006).

(b) Without knowledge of the results from the Pattern Glare Test above, measurements of reading speed with and without overlays were conducted by a second experimenter. The Intuitive Overlay system (i.O.O. Sales, London) was used. The Intuitive Overlays comprise a set of coloured overlays that have been designed to sample colours systematically and which are of an adequate size to be effective (Waldie and Wilkins 2004) and have a sufficient range of colours (Smith and Wilkins 2007). The set consists of 20 A5-sized overlays (2 sets of 10 different coloured overlays). Following the procedure recommended in the manual, all eighty participants chose from the Intuitive Overlays the colour of overlay that best improved the clarity and comfort of the text it covered (one of two passages of randomly ordered common words, arranged side by side). The procedures followed a forced choice procedure whereby each participant had to compare the two texts, one or both of which were covered by an overlay, and choose which text was more comfortable to view. Note that for the purposes of control, all participants were forced to make such a choice whether or not they reported a benefit from the overlay. Figure 1 shows the chromaticities of the overlays chosen by both groups.

The Rate of Reading Test (Wilkins et al 1996) was administered four times: first with, then without, then again without, and finally with their chosen overlay. The purpose of the ABBA design was to minimise practice effects. Most of the practice effects occur from the first to the second administration, and the design therefore biases any mean difference against a benefit. There is no evidence that individuals who read faster do so because they tolerate a greater number of errors (Wilkins et al 1996). An average rate of reading with and without the overlay was calculated, along with the percentage difference between the two conditions. 


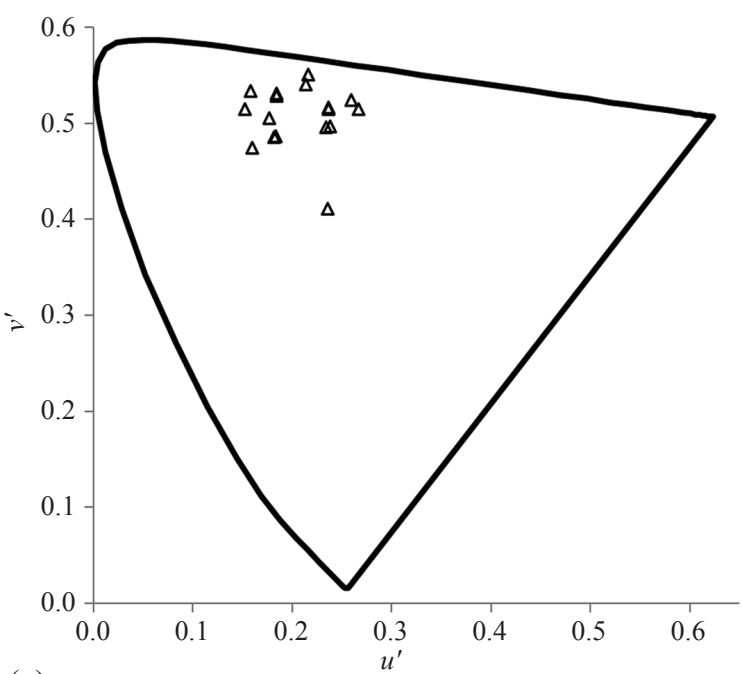

(a)

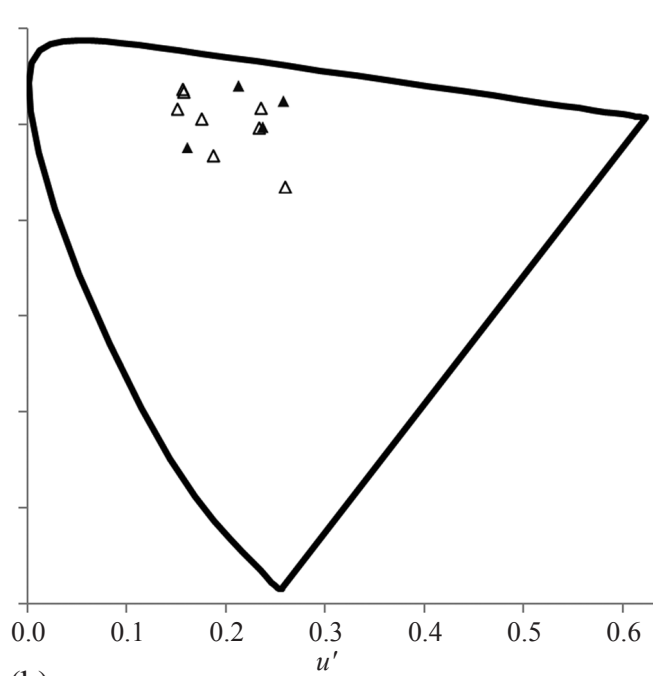

(b)

Figure 1. CIE 1976 uniform chromaticity scale diagrams showing the $u^{\prime} v^{\prime}$ chromaticities of the light viewed by participants when observing the chosen coloured overlays through the optics of the autorefractor. The filled points indicate the chromaticities of overlays chosen by more than one participant. (a) PRVS participants; (b) control participants.

An improvement of more than 5\% in reading speed on the Wilkins Rate of Reading Test with the chosen coloured overlay has been associated with clinical benefit as expressed by sustained voluntary use (Jeanes et al 1997).

In order to classify a participant as susceptible to PRVS both a pattern glare score of 3 or more and an improvement in reading speed of more than 5\% with their chosen overlay was required, so as to sample the extremes of the susceptibility within the cohort of eighty participants. This resulted in eighteen participants being classified as susceptible to PRVS and eighteen matched participants in the control group with scores below criterion on both pattern glare and reading speed increment.

Although pattern glare scores and rate of reading scores correlated positively across the group ( $r=0.67 ; p<0.001)$, the selection procedure resulted in the exclusion of (i) nine participants with pattern scores of 3 or more whose rate of reading scores did not exceed the $5 \%$ criterion and (ii) sixteen participants whose rate of reading scores exceeded the $5 \%$ criterion but whose pattern glare score was less than 3 .

The mean ages $( \pm$ SD) for the PRVS and control groups were respectively $20.9( \pm 2.4)$ years and $20.7( \pm 2.2)$ years. The mean spherical equivalent refractive error for the two groups was PRVS-RE -2.4 ( \pm 2.8$)$ D LE -2.3 ( \pm 3.0$)$ D; controls-RE -2.2 ( \pm 3.1$)$ D LE $-2.3( \pm 3.1)$ D. The near point of convergence values were similar: PRVS $-6.0( \pm 1.5) \mathrm{cm}$; control -6.7 $( \pm 1.7) \mathrm{cm}$. In summary, the two groups, PRVS and control, were matched with respect to age, refractive error, and near point of convergence, and no participant had colour vision deficiency. Every participant had an amplitude of accommodation (measured subjectively with a RAF rule) greater than $10 \mathrm{D}$, so none was excluded on the basis of accommodative insufficiency. The groups differed in that the PRVS group was subject to pattern-related visual stress and read more quickly with an overlay of their chosen colour.

\subsection{Session 2: Measurements of accommodation and comfort under various conditions}

Two further experimenters conducted the investigations in session 2 without knowledge of the findings obtained in session 1, or the allocation of participants. The doublemasked design ensures that the relationships between the variables cannot be attributed to experimenter bias. 


\subsection{Assessment of accommodative response}

The accommodative response was measured with a Shin-Nippon SRW-5000 open field autorefractor. The autorefractor (Ajinomoto Trading, Tokyo, Japan) allows an objective measure of accommodative response while participants binocularly view in an unenclosed environment. Mallen et al (2001) showed the Shin-Nippon SRW-5000 autorefractor was highly correlated with subjective refraction and repeatable over the prescription range used during accommodation studies.

All participants were corrected using spherical contact lenses (1-day Acuvue Moist by Johnson \& Johnson) to within $0.25 \mathrm{D}$. In order to ensure all participants were optimally corrected (confirmed with a spherical contact lens over-refraction), any small residual spherical refractive errors were corrected, where necessary, with trial lenses; the maximum additional trial lens used was $0.25 \mathrm{DS}$. This was necessary for only two participants (one from each group).

5 measurements of accommodation were taken with viewing at $0.4 \mathrm{~m}$ the grey square ( $0.3 \mathrm{deg})$ at the centre of a horizontal square-wave grating, spatial frequency $2.2 \mathrm{cycles} \mathrm{deg}^{-1}$; subtending $14.3 \mathrm{deg}$ at the eye. The 5 measurements were averaged and converted into spherical equivalents (sphere power plus half cylindrical power). The viewing conditions were as follows:

(a) target,

(b) target with overlay of chosen colour,

(c) target with $+0.75 \mathrm{D}$ trial lenses,

(d) target with overlay and $+0.75 \mathrm{D}$ trial lenses,

(e) target with $-0.75 \mathrm{D}$ lenses,

(f) target with overlay and $-0.75 \mathrm{D}$ lenses.

The order of presentation for each participant was randomised. To calculate the accommodative lag, the accommodative response was subtracted from the various accommodative demands above.

The participants viewed the stimuli binocularly, although accommodation measurements were taken from the right eye only. The experimenter ensured optimum positioning of the right eye in line with the autorefractor. The convergence required to fixate the grey square was approximately $7 \mathrm{deg}$. This is within the $10 \mathrm{deg}$ tolerance of the Shin-Nippon SRW-5000 open field autorefractor (Wolffsohn et al 2002).

\subsection{Assessment of pattern glare}

Pattern glare scores were collected when viewing at $0.4 \mathrm{~m}$ :

(a) the Pattern Glare Test (as used above),

(b) the Pattern Glare Test viewed through a pair of $+0.75 \mathrm{D}$ lenses,

(c) the Pattern Glare Test viewed through a pair of $-0.75 \mathrm{D}$ lenses.

The order of presentation for each participant was randomised.

\section{Results}

The selection of participants resulted in two groups. In the PRVS group the mean (SD) increase in rate of reading (words per minute) with the overlay of chosen colour was $10.4 \pm 4.8$ and the mean pattern glare score was $4.8 \pm 1.7$. In the matched control group the scores were respectively $-1.1 \pm 4.7$ and $0.7 \pm 0.8$.

Figure 2 shows the mean and standard deviation of the accommodative lag while viewing the grating of the Pattern Glare Test, for the PRVS and matched control groups, with no overlay, with chosen overlay, and with and without $+0.75 \mathrm{D}$ and $-0.75 \mathrm{D}$ lenses. 


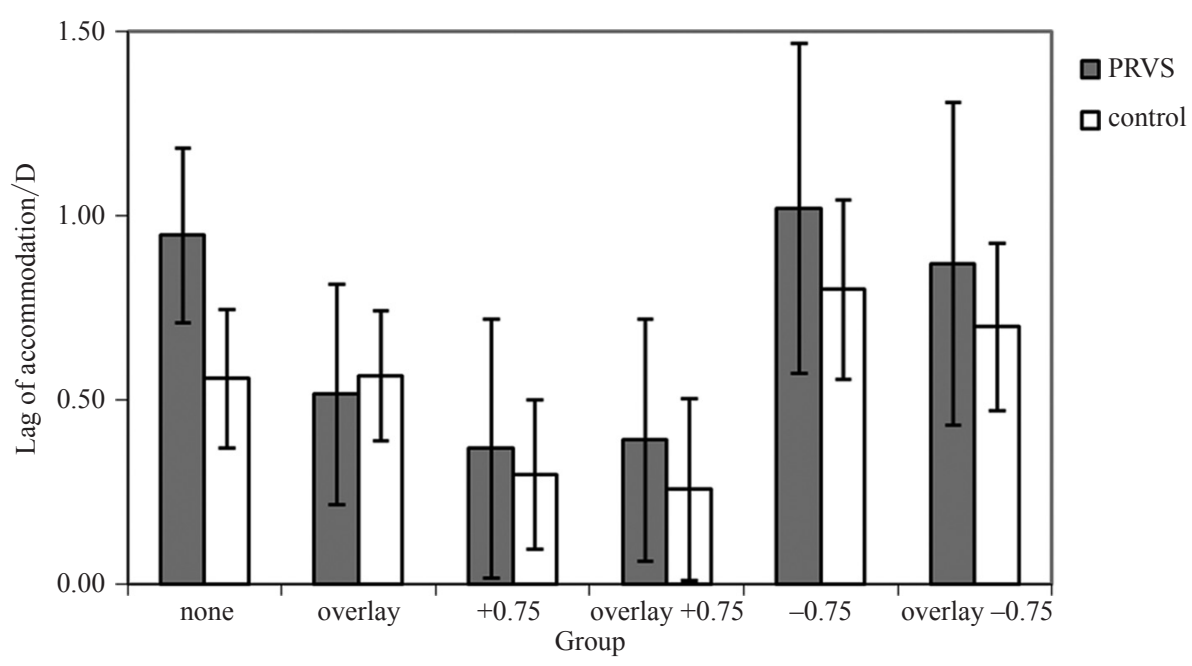

Figure 2. The mean accommodative lag while viewing the grating of the Pattern Glare Test, for the PRVS and matched control groups, with no overlay, with chosen overlay, and with and without $+0.75 \mathrm{D}$ and $-0.75 \mathrm{D}$ lenses. Error bars represent standard deviation.

\subsection{Effect of lenses}

A mixed repeated-measures ANOVA of the data obtained without lenses and with overlay as the within-subject factor and group as the between-subject factor revealed a significant main effect of the presence of an overlay $\left(F_{1,34}=34.62, p<0.0001\right)$ and a significant interaction term $\left(F_{1,34}=37.21, p<0.0001\right)$. As expected from previous work (Allen et al 2010b), the accommodative lag was greater in the PRVS group. The interaction term occurred because, again as expected (Allen et al 2010b), the accommodative lag was significantly reduced with an overlay of chosen colour in the PRVS group $\left(t_{17}=6.97, p<0.0001\right)$ but not in the control group $\left(t_{17}=0.21, p=0.83\right)$.

The above findings raise the possibility that the failure to observe an effect of the overlay on accommodative lag in the control group might be due, at least in part, to their lower habitual lag of accommodation. This possibility was explored by assessing the effect of the overlay when the accommodative lag in the control group was increased with negative lenses, which had the effect of increasing the lag. A repeated-measures ANOVA of the data for the control group with the presence of overlay and of negative lenses as separate factors revealed a significant main effect of the lenses $\left(F_{1,17}=11.7, p=0.003\right)$, no significant effect of the overlay $\left(F_{1,17}=2.52, p=0.13\right)$, and no interaction term $\left(F_{1,17}=2.86, p=0.11\right)$. There therefore remained no effect of the overlay despite a significant effect of the negative lenses in increasing accommodative lag.

The addition of negative lenses may have reduced any effect of an overlay by taking the accommodative lag beyond the individuals' habitual limits. However, in the PRVS group, the overlay remained effective when the negative lenses increased the accommodative lag still further. This was demonstrated by a repeated-measures ANOVA of the data for the PRVS group with/without overlays and with/without negative lenses. There was a main effect of the negative lenses $\left(F_{1,17}=6.62, p=0.02\right)$ and overlay $\left(F_{1,17}=30.72, p<0.001\right)$, and a significant interaction term $\left(F_{1,17}=13.88, p=0.002\right)$. The interaction could be attributed to a smaller difference between accommodative lags with and without the overlay when the negative lenses were worn $\left(t_{17}=3.72, p=0.002\right)$.

The PRVS group had a large accommodative lag. When the lag of accommodation was reduced by positive lenses there was no significant change in the lag with the addition of the overlay. This was confirmed by a repeated-measures ANOVA with presence/absence of overlay and positive lenses as factors, which revealed a significant effect of the lenses in 
reducing the accommodative lag $\left(F_{1,17}=23.85, p<0.001\right)$, a significant effect of overlays $\left(F_{1,17}=23.51, p<0.001\right)$, and a significant interaction term $\left(F_{1,17}=48.91, p<0.001\right)$. The interaction was attributable to an effect of the overlay when no (positive) lenses were worn $\left(t_{17}=6.97, p<0.001\right)$, but not when the lenses were worn $\left(t_{17}=0.53, p=0.60\right)$.

\subsection{Pattern glare}

Table 2 shows the number of illusions on pattern 2 of the Pattern Glare Test, separately for the PRVS and matched control group and the three experimental conditions that did not involve a coloured filter.

Table 2. Number of illusions on pattern 2 of the Pattern Glare Test, shown separately for the PRVS and matched control group and the experimental conditions. The data are for trials on which no overlay was used. Numbers in parentheses are standard deviations.

\begin{tabular}{llll}
\hline Group & No lenses & Positive lenses & Negative lenses \\
\hline PRVS & $4.8(1.7)$ & $3.4(2.0)$ & $2.9(1.9)$ \\
Control & $0.7(0.8)$ & $0.7(1.0)$ & $0.6(0.9)$ \\
\hline
\end{tabular}

A mixed repeated-measures ANOVA of the three lens conditions without the overlay showed the expected effect of group $\left(F_{1,34}=54.77, p<0.0001\right)$, a significant main effect of lens condition $\left(F_{2,68}=10.12, p<0.0001\right)$, and a significant interaction term $\left(F_{2,68}=8.10\right.$, $p=0.0007)$. The interaction term was explained by the fact that in the PRVS group there was a reduction in pattern glare with lenses of either power $\left(t_{17}=2.990, p=0.01\right.$, positive lens; $t_{17}=3.99, p<0.001$, negative lens), whereas there were no such effects in the control group. The reduction is possibly the result of demand characteristics: participants with symptoms expecting a lens to reduce them, and for this reason the effects of accommodative lag on pattern glare were studied with an ANOVA of the two lens conditions alone. This analysis showed a main effect of group $\left(F_{1,34}=27.58, p<0.001\right)$, but no main effect of the power of lenses $\left(F_{1,34}=2.16, p=0.15\right)$ and no significant interaction term $\left(F_{1,34}=1.31, p=0.26\right)$. Although both lenses reduced pattern glare, there was no effect of the sign of the lens power.

\section{Discussion}

The PRVS group had larger lags of accommodation, and the chosen overlay reduced the lag, replicating our previous findings (Allen et al 2010b). There was no effect of the overlay on lag of accommodation in the control group, again as we previously found (Allen et al 2010b). Here we have shown that the absence of such an effect could not be attributed simply to the generally lower lags of accommodation in the control group because there remained no effect of overlays when the lags of accommodation were increased with negative lenses to levels that were similar to those in the PRVS group.

Because of the selection criteria, pattern glare scores were greater in the PRVS group than the controls. In both the control group and the PRVS group, pattern glare scores were unaffected by the sign of the power of the trial lenses. This finding suggests the possibility that symptoms of pattern glare are unrelated to accommodation in any systematic way, in accordance with previous work in which there was no difference in accommodative lag between a stressful and non-stressful target (Allen et al 2010b) and possibly because demand characteristics due to the presence of a lens were masking any effects. In future work, the use of lenses of zero power will clarify this issue. The aspects of accommodation and the sequence of events leading to a focussed image are complex, as they involve sensory, motor, neurological, anatomical, biomechanical, and perceptual components (Mordi and Ciuffreda 2004). Voluntary accommodation may represent a pre-programmed manoeuvre used in a variety of predictable situations to optimise performance (Ciuffreda and Kruger 
1988). Provine and Enoch (1975) suggested that in some people voluntary accommodative effort provides an initial accommodative movement in the correct direction. If subjective experiences of pattern glare influence voluntary accommodative effort, then it is difficult to explain the above findings in terms of such effort.

The viewing conditions were binocular, so manipulation of accommodation will have been associated with changes of vergence. Nevertheless, the manipulation of accommodative lag using lenses increases and decreases the lag as expected, and does so in similar ways in both PRVS and control groups, though to a greater extent in the PRVS group, possibly because of their larger habitual lag. It is possible that participants could have attempted to exercise voluntary accommodation in an attempt to compensate for any induced heterophoria using accommodative vergence (Burian 1945). Alternatively, the demand characteristics of the trial lenses may have reduced the report of some of the pattern glare symptoms, masking the differences between lenses.

Evans (2001) noted controversy whether convergence insufficiency is a correlate of dyslexia. Convergence insufficiency is a prevalent condition and is encountered quite commonly in good and poor readers. Although only one study since 2001 has found a slightly more remote near point of convergence in a group of children with dyslexia than in a control group (Kapoula and Bucci 2007), we wanted to ensure it did not influence the accommodation findings in this study and so we matched the groups.

Longitudinal chromatic aberration (LCA) provides one possible explanation for any relationship between colour and accommodation. Chase et al (2007) reported psychophysical findings suggesting that, as L/M cone contrast sensitivity increased, reading performance decreased. Several studies have found that accommodative demand may be influenced by the L/M cone contrast ratio through the mechanism of LCA (Kruger et al 1995; Lee et al 1999; Stark et al 2002; Rucker and Kruger 2006). Chase et al (2007) proposed that selecting colours that reduce LCA-induced accommodative demand may be one way to improve focus and reduce symptoms. Such an explanation, however, would predict a benefit from coloured filters once the accommodative lag in the control group was increased to levels similar to those in the PRVS group, and that did not occur. It is possible that the choice of coloured filters by the control group might differ (and be more appropriate) when such a lag was present. Future studies will investigate LCA in relation to colour choice and accommodative lag.

The hypothesis of cortical hyperexcitability (Wilkins 1995) provides another explanation. If the text is found to be uncomfortable to readers who are susceptible to pattern glare because of cortical over-activation (Wilkins et al 1984), then blur would reduce such activation owing to reduction in the aversiveness as a consequence of contrast reduction, although any such reduction would be small. If colour reduces over-activation, then a reduced lag of accommodation may result (Allen et al 2010b).

When gratings are observed, the degrading effect of defocus blur on the contrast of the retinal image increases with the spatial frequency of the grating, although the exact contrast changes vary with changes in pupil diameter, wavelength, and ocular aberrations (Green and Campbell 1965; Charman and Jennings 1976; Charman 1979; Atchison et al 1998; Marcos et al 1999; Taylor et al 2009).

Charman and Tucker (1978) and Charman (1979) found accommodative accuracy was maintained or increased at high spatial frequencies whereas Owens (1980) found maximal accuracy of accommodation at 3-5 cycles $\mathrm{deg}^{-1}$ with accuracy decreasing at higher and lower frequencies. Differences may be due to instructions (Stark and Atchison 1994). Charman and Tucker encouraged maximal use of voluntary accommodation, whereas Owen's participants were instructed to view naturally, as in the present study, relying mainly on reflex accommodation.

The present findings support previous work but indicate that symptoms of pattern glare are not strongly associated with accommodation. 


\section{References}

Allen P M, Evans B J W, Wilkins A J, 2010a Vision and Reading Difficulties (London: Ten Alps)

Allen P M, Hussain A, Usherwood C, Wilkins A J, 2010b "Pattern-related visual stress, chromaticity and accommodation" Investigative Ophthalmology \& Visual Science 51 6843-6849

Allen P M, Radhakrishnan H, O’Leary D J, 2003 “Repeatability and validity of the PowerRefractor and the Nidek A R600-A in an adult population with healthy eyes" Optometry and Vision Science $80245-251$

Atchison D A, Charman W N, Woods R L, 1997 "Subjective depth-of-focus of the eye" Optometry and Vision Science 74 511-520

Atchison D A, Woods R L, Bradley A, 1998 "Predicting the effects of optical defocus on human contrast sensitivity" Journal of the Optical Society of America 15 2536-2544

Burian H M, 1945 "Intermittent (facultative) divergence strabismus. Its influence on visual acuity and the binocular activity" American Journal of Ophthalmology 28 525-527

Charman W N, 1979 "Effect of refractive error in visual tests with sinusoidal gratings" British Journal of Physiological Optics 33 10-20

Charman W N, Jennings J A, 1976 "The optical quality of the monochromatic retinal image as a function of focus" British Journal of Physiological Optics 31 119-134

Charman W N, Tucker J, 1978 "Accommodation as a function of object form" American Journal of Optometry and Physiological Optics 65 84-92

Chase C, Dougherty R F, Ray N, Fowler S, Stein J, 2007 "L/M speed matching ratio predicts reading in children" Optometry \& Vision Science 84 229-236

Chase C, Tosha C, Borsting E, Ridder W H, 2009 "Visual discomfort and objective measures of static accommodation" Optometry and Vision Science 86 883-889

Ciuffreda K J, Kruger P B, 1988 "Dynamics of human voluntary accommodation" American Journal of Optometry and Physiological Optics 65 365-370

Ciuffreda K J, Scheiman M, Ong E, Rosenfield M, Solan H A, 1997 "Irlen lenses do not improve accommodative accuracy at near" Optometry \& Vision Science 74 298-302

Evans B J W, 2001 Dyslexia and Vision (Chichester: John Wiley)

Evans B J W, Stevenson S J, 2008 "The Pattern Glare Test: a review and determination of normative values" Ophthalmic \& Physiological Optics 28 295-309

Green D G, Campbell F W, 1965 "Effect of defocus on the visual response to a sinusoidally modulated stimulus" Journal of the Optical Society of America 55 1154-1157

Harle D E, Shepherd A J, Evans B J W, 2006 "Visual stimuli are common triggers of migraine and are associated with pattern glare" Headache 46 1431-1440

Hollis J, Allen P M, 2006 "Screening for Meares-Irlen sensitivity in adults: Can assessment methods predict changes in reading speed?" Ophthalmic \& Physiological Optics 26 566-571

Jeanes R, Busby A, Martin J, Lewis E, Stevenson N, Pointon D, Wilkins, A J, 1997 "Prolonged use of coloured overlays for classroom reading" British Journal of Psychology 88 531-548

Kapoula Z, Bucci M P, 2007 "Postural control in dyslexic and non-dyslexic children" Journal of Neurology 254 1174-1183

Karania R, Evans B J, 2006 "The Mallett Fixation Disparity Test: influence of test instructions \& relationship with symptoms" Ophthalmic \& Physiological Optics 26 507-522

Kruger P B, Mathews S, Aggarwala K R, Yager D, Kruger E S, 1995 "Accommodation responds to changing contrast of long, middle and short spectral-waveband components of the retinal image" Vision Research 35 2415-2429

Lee J H, Stark L R, Cohen S, Kruger P B, 1999 "Accommodation to static chromatic simulations of blurred retinal images" Ophthalmic \& Physiological Optics 19 223-235

Mallen E A, Wolffsohn J S, Gilmartin B, Tsujimura S, 2001 "Clinical evaluation of the Shin-Nippon SRW-5000 autorefractor in adults" Ophthalmic \& Physiological Optics 21 101-107

Marcos S, Moreno E, Navarro R, 1999 "The depth-of-field of the human eye from objective and subjective measurements" Vision Research 39 2039-2049

Mordi J A, Ciuffreda K J, 2004 "Dynamic aspects of accommodation: age and presbyopia" Vision Research 44 591-601 
Nulty D, Wilkins A J, Williams J M, 1987 "Mood, pattern sensitivity and headache: a longitudinal study" Psychological Medicine 17 705-713

Owens D A, 1980 "A comparison of accommodative responsiveness and contrast sensitivity for sinusoidal gratings" Vision Research 20 159-167

Provine R R, Enoch J M, 1975 “On voluntary ocular accommodation” Perception \& Psychophysics 17 209-212

Rouse M W, Hutter R F, Shiftlett R, 1984 “A normative study of the accommodative lag in elementary school children" American Journal of Optometry and Physiological Optics 61 693-699

Rucker F J, Kruger P B, 2006 "Cone contributions to signals for accommodation and the relationship to refractive error" Vision Research 46 3079-3089

Simmers A J, Gray L S, Wilkins A J, 2001 "The influence of tinted lenses upon ocular accommodation" Vision Research 41 1229-1238

Smith L, Wilkins A J, 2007 "How many colours are necessary to increase the reading speed of children with visual stress? A comparison of two systems" Journal of Research in Reading 30 332-343

Stark L R, Atchison D A, 1994 "Subject instructions and methods of target presentation in accommodation research" Investigative Ophthalmology \& Visual Science 35 528-537

Stark L R, Lee R S, Kruger P B, Rucker F J, Ying Fan H, 2002 “Accommodation to simulations of defocus and chromatic aberration in the presence of chromatic misalignment" Vision Research $\mathbf{4 2}$ $1485-1498$

Taylor J, Charman W N, O’Donnell C, Radhakrishnan H, 2009 "Effect of target spatial frequency on accommodative response in myopes and emmetropes" Journal of Vision 9(1):16, 1-14

Tosha C, Borsting E, Ridder W H, Chase C, 2009 "Accommodation response and visual discomfort" Ophthalmic \& Physiological Optics 29 625-633

Waldie M, Wilkins A J, 2004 "How big does a coloured overlay have to be?" Ophthalmic \& Physiological Optics 24 57-60

Wilkins A J, 1995. Visual Stress (Oxford: Oxford University Press)

Wilkins A J, Evans B J W, 2001 Pattern Glare Test Instructions (London: i.O.O. Sales)

Wilkins A J, Jeanes R J, Pumfrey P D, Laskier M, 1996 "Rate of Reading Test: its reliability, and its validity in the assessment of the effects of coloured overlays" Ophthalmic \& Physiological Optics $16491-497$

Wilkins A J, Nimmo-Smith M I, Tait A, McManus C, Della Sala S, Tilley A, Arnold K, Barrie M, Scott S, 1984 "A neurological basis for visual discomfort" Brain 107 989-1017

Wolffsohn J S, Hunt O A, Gilmartin B, 2002 "Continuous measurement of accommodation in human factor applications" Ophthalmic \& Physiological Optics 22 380-384 